\title{
Pharmacological activities, mechanisms of action, and safety of salidroside in the central nervous system
}

This article was published in the following Dove Press journal:

Drug Design, Development and Therapy

\author{
Zhifeng Zhong' \\ Jing $\operatorname{Han}^{\prime}$ \\ Jizhou Zhang' \\ Qing Xiao' \\ Juan $\mathrm{Hu}^{1,2}$ \\ Lidian Chen ${ }^{1,2}$ \\ 'Institute of Materia Medica, Fujian \\ Academy of Traditional Chinese \\ Medicine, Fuzhou, Fujian, People's \\ Republic of China; ${ }^{2}$ School of \\ Rehabilitation Medicine, Fujian \\ University of Traditional Chinese \\ Medicine, Fuzhou, Fujian, People's \\ Republic of China
}

\begin{abstract}
The primary objective of this review article was to summarize comprehensive information related to the neuropharmacological activity, mechanisms of action, toxicity, and safety of salidroside in medicine. A number of studies have revealed that salidroside exhibits neuroprotective activities, including anti-Alzheimer's disease, anti-Parkinson's disease, antiHuntington's disease, anti-stroke, anti-depressive effects, and anti-traumatic brain injury; it is also useful for improving cognitive function, treating addiction, and preventing epilepsy. The mechanisms underlying the potential protective effects of salidroside involvement are the regulation of oxidative stress response, inflammation, apoptosis, hypothalamus-pituitary-adrenal axis, neurotransmission, neural regeneration, and the cholinergic system. Being free of side effects makes salidroside potentially attractive as a candidate drug for the treatment of neurological disorders. It is evident from the available published literature that salidroside has potential use as a beneficial therapeutic medicine with high efficacy and low toxicity to the central nervous system. However, the definite target protein molecules remain unclear, and clinical trials regarding this are currently insufficient; thus, guidance for further research on the molecular mechanisms and clinical applications of salidroside is urgent.
\end{abstract}

Keywords: salidroside, Alzheimer's disease, Parkinson's disease, stroke, cognitive impairment, clinical trials

\section{Introduction}

Rhodiola rosea L., a small genus of the Crassulaceae family, has a long history of wide use as a botanical medicine in Europe, Asia, and the US to prevent and treat a great variety of common conditions and complex diseases, including fatigue, various pains, Alzheimer's disease (AD), depression, and anxiety. ${ }^{1,2}$ It is also used as an adaptogen and cardiopulmonary protective agent in traditional folk medicine. ${ }^{3}$ Rhodiola rosea extracts contain about $1 \%$ salidroside (Figure 1). ${ }^{4}$ The salidroside content contributes a lot to the properties of Rhodiola, and is the main active bioactive component in Rhodiola rosea. Salidroside has also been found in other species. ${ }^{5-10}$ Meanwhile, the chemical and biological synthesis of salidroside have been investigated in various studies. ${ }^{11-16}$

In pharmacokinetics, $p$-tyrosol (aglycone of salidroside) has been identified as a metabolite in the plasma of rats treated with salidroside, and salidroside was detected in brain tissue. ${ }^{17-19}$ Some studies have reported that salidroside has various pharmacological effects, such as an anti-AD, anti-PD, anti-stroke, anti-Huntington's disease (HD), anti-TBI, anti-depressive, anti-cancer, anti-coxsackie, rejuvenating, anti-diabetes, cardioprotective, vasculoprotective, and hepatoprotective effects, as
Correspondence: Lidian Chen; Juan Hu Institute of Materia Medica, Fujian Academy of Traditional Chinese Medicine, Number 282 Wusi Road, Gulou, Fuzhou 350003, Fujian,

People's Republic of China Email cld@fjtcm.edu.cn; huj@fjtcm.edu.cn (c) (1) (2) $2018 \mathrm{Zhong}$ et al. This work is published and licensed by Dove Medical Press Limited. The full terms of this license are available at https://www.dovepress.com/terms.php
and incorporate the Creative Commons Attribution - Non Commercial (unported, v3.0) License (http://creativecommons.org/licenses/by-nc/3.0/). By accessing the work you hereby accept the Terms. Non-commercial uses of the work are permitted without any further permission from Dove Medical Press Limited, provided the work is properly attributed. For permission for commercial use of this work, please see paragraphs 4.2 and 5 of our Terms (https://www.dovepress.com/terms.php). 


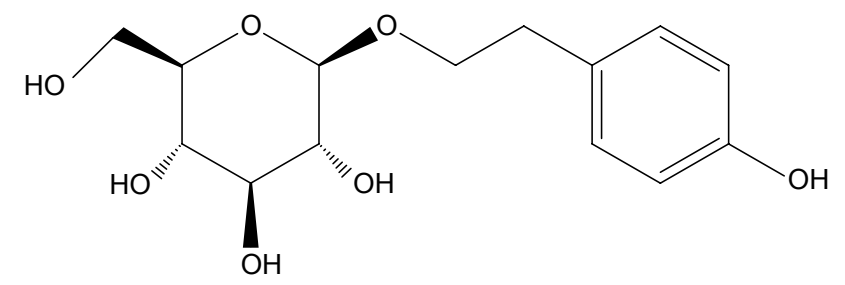

Figure I Chemical structure of salidroside, 2-(4-hydroxyphenyl) ethyl- $\beta$-Dglucopyranoside (molecular formula: $\mathrm{C}_{14} \mathrm{H}_{20} \mathrm{O}_{7}$; molecular weight: $300.3 \mathrm{I}$; CAS registry number: 10338-5I-9; PubChem CID: I59278).

Abbreviations: CAS, Chemical Abstracts Service; CID, Compound Identifier.

well as improves cognitive function, treats addiction, and prevents epilepsy across a wide therapeutic time window. ${ }^{19-32}$

Recently, an increasing number of studies have been conducted on the neuroprotective effects of salidroside (Figure 2); however, neither in vivo nor in vitro experiments have been thoroughly studied. This review will systematically summarize and comprehensively analyze the developments and adaptogenic mechanisms of action in the neuropharmacological study of salidroside (Table 1; Figure 3), and provide suggestions for further studies.

\section{Pharmacological activity and mechanisms of action}

\section{Anti-Alzheimer's disease effect}

$\mathrm{AD}$, the most common progressive neurodegenerative disorder, is clinically characterized by cognitive impairment, memory dysfunction, and behavioral disorder in the elderly. AD affects approximately 36 million people worldwide, and this number is expected to nearly double over the next 20 years. $^{33,34}$ Drug treatment is the most commonly used option for the disease. At present, only a small number of drugs have been approved in clinical practice, such as donepezil, rivastigmine, galantamine, memantine, and the
Chinese herb extract huperzine A. ${ }^{35,36}$ Additional traditional Chinese medicine studies have shown that salidroside exerts promising effects in the treatment of AD.

In recent years, an increasing body of evidence has suggested that $\mathrm{A} \beta$ plays an important role in neuronal dysfunction and cellular death. ${ }^{37}$ Thus, $A \beta$-induced disease is an important research focus for examining $\mathrm{AD}$ onset and progression. An in vitro study in $\mathrm{A} \beta_{1-42}$-induced AD PC12 cell lines determined that salidroside reduced cytotoxicity, attenuated reactive oxygen species (ROS) accumulation, and decreased intracellular malondialdehyde by activating antioxidant enzymes in a dose-dependent manner after $3 \mathrm{~h}$ pre-incubation with salidroside $(1,5,10$, and $50 \mathrm{mg} / \mathrm{mL}){ }^{38}$ Furthermore, pretreatment with salidroside $(10,50$, and $100 \mu \mathrm{M}$ ) was shown to significantly inhibit oxidative stress and apoptosis in $\mathrm{A} \beta_{25-35}$-induced SH-SY5Y cells in a concentration-dependent manner in vitro via increasing antioxidant enzyme activities, modulating apoptosis-related protein expression, and restoring anomalies in the mitochondrial membrane potential (MMP) and ROS production. ${ }^{39}$ In addition, it was found that salidroside $(5,100$, and $200 \mu \mathrm{M})$ decreased $A \beta$ levels in $A \beta_{1-42}$-incubated primary neurons via $\mathrm{PI} 3 \mathrm{~K} / \mathrm{Akt} / \mathrm{mTOR}$ signaling. ${ }^{40}$ Salidroside $(2,6$, and $20 \mu \mathrm{M}$ ) also improved both the longevity and locomotor activity of Tao transgenic Drosophila in a dose-dependent manner. These studies revealed that the protective effects of salidroside were due to the up-regulation of total p-GSK3 $\beta$ and down-regulation of p-tau. ${ }^{40,41}$

Apart from the A $\beta$-induced AD model streptozotocin (STZ), D-galactose, glutamate, $\mathrm{H}_{2} \mathrm{O}_{2}$, and hypoxia are also used to mimic $\mathrm{AD}$ in studies. A previous study revealed that direct incubation with salidroside $(1 \mathrm{mM})$ exhibited neuroprotective effects on active neural stem cells (NSCs)

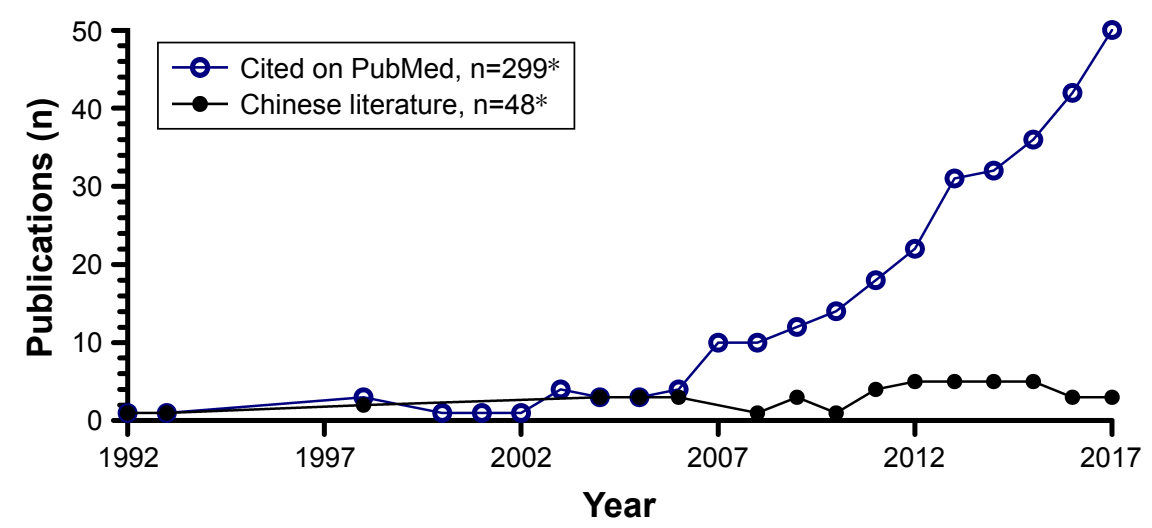

Figure 2 Salidroside has been receiving increasing interest in the scientific community. In total, about 300 scientific publications on salidroside can be found in the literature from 1992 to 2017.

Note: *References from PubMed. 


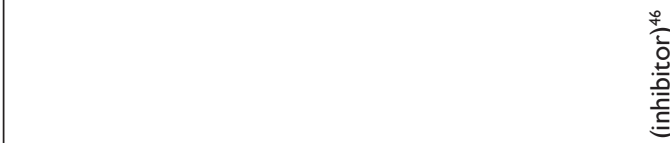

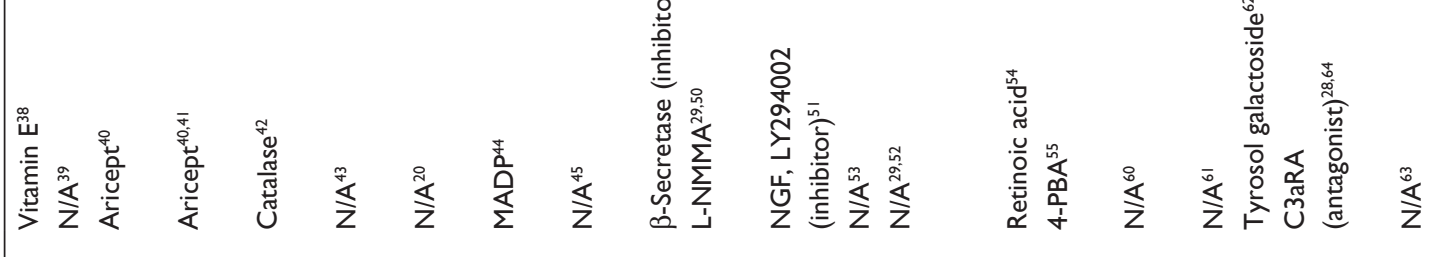

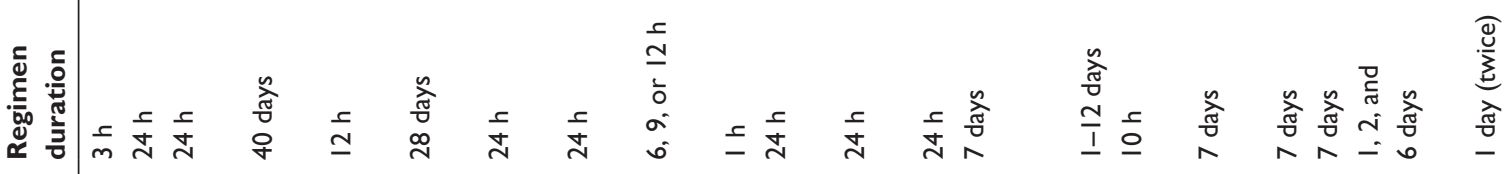

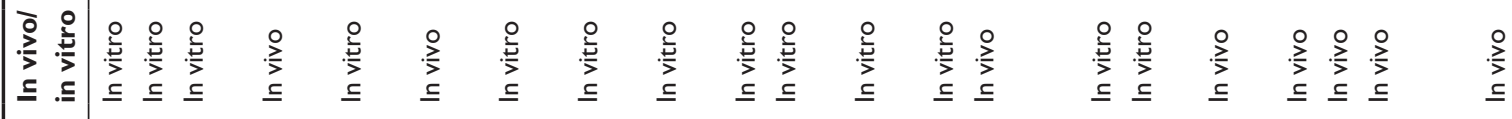

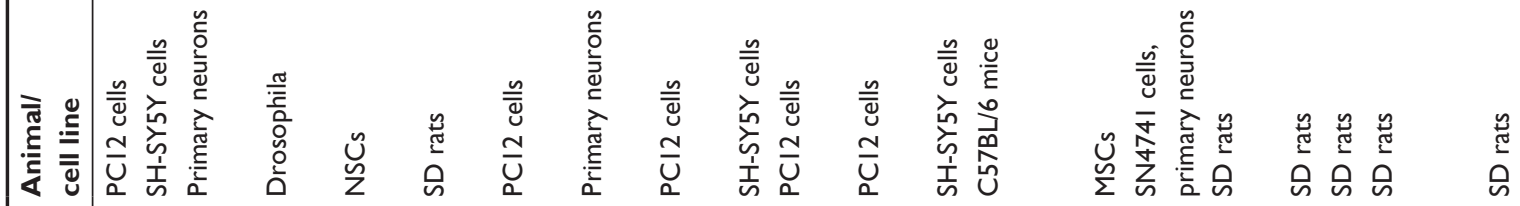

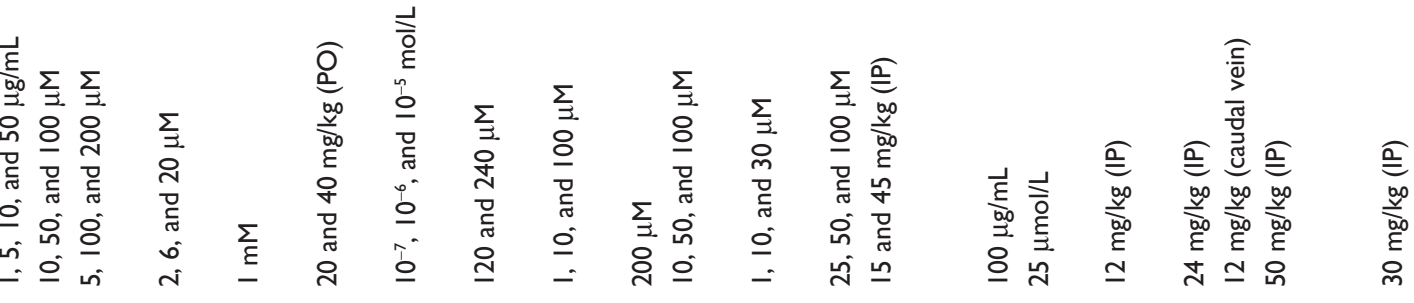

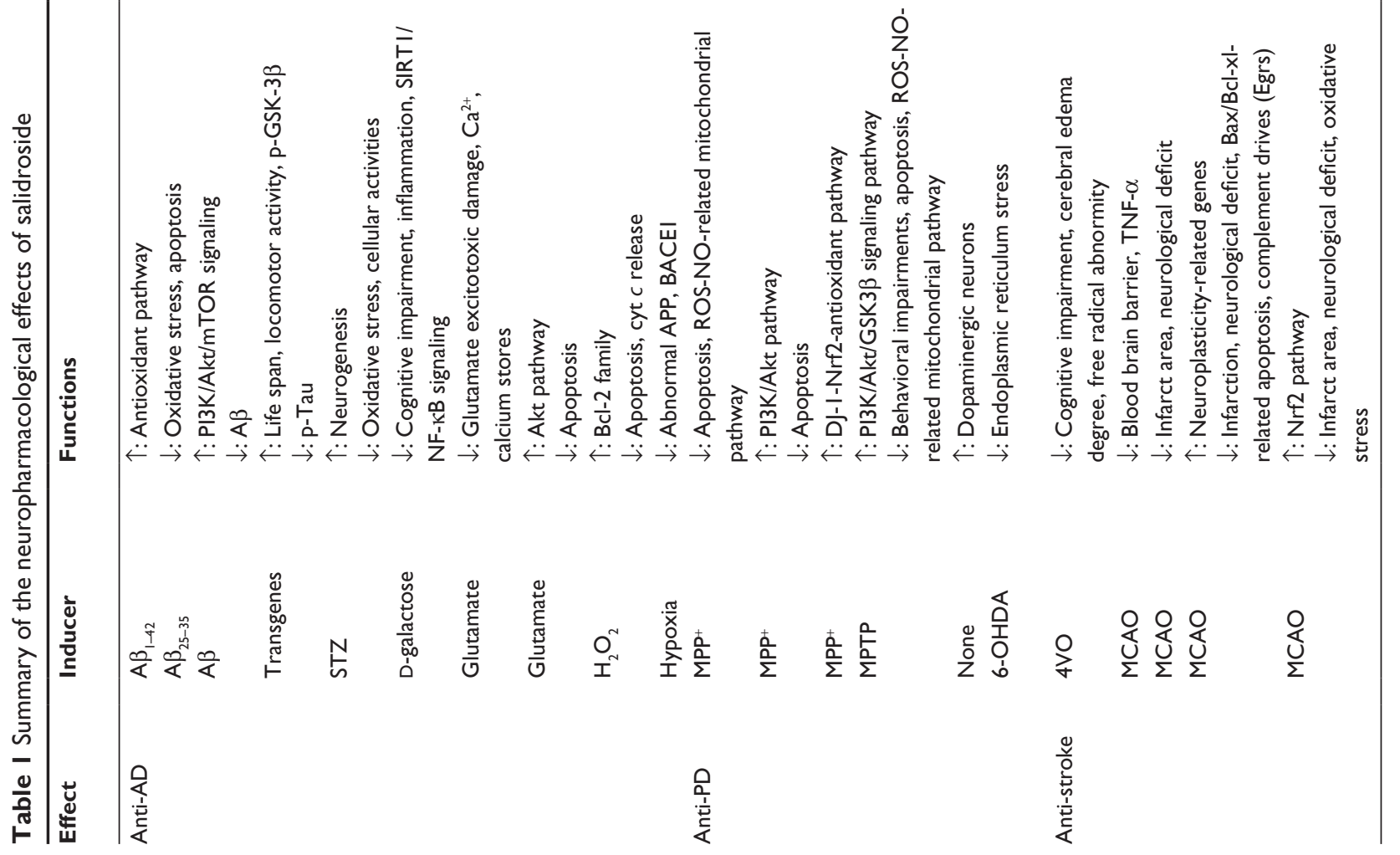




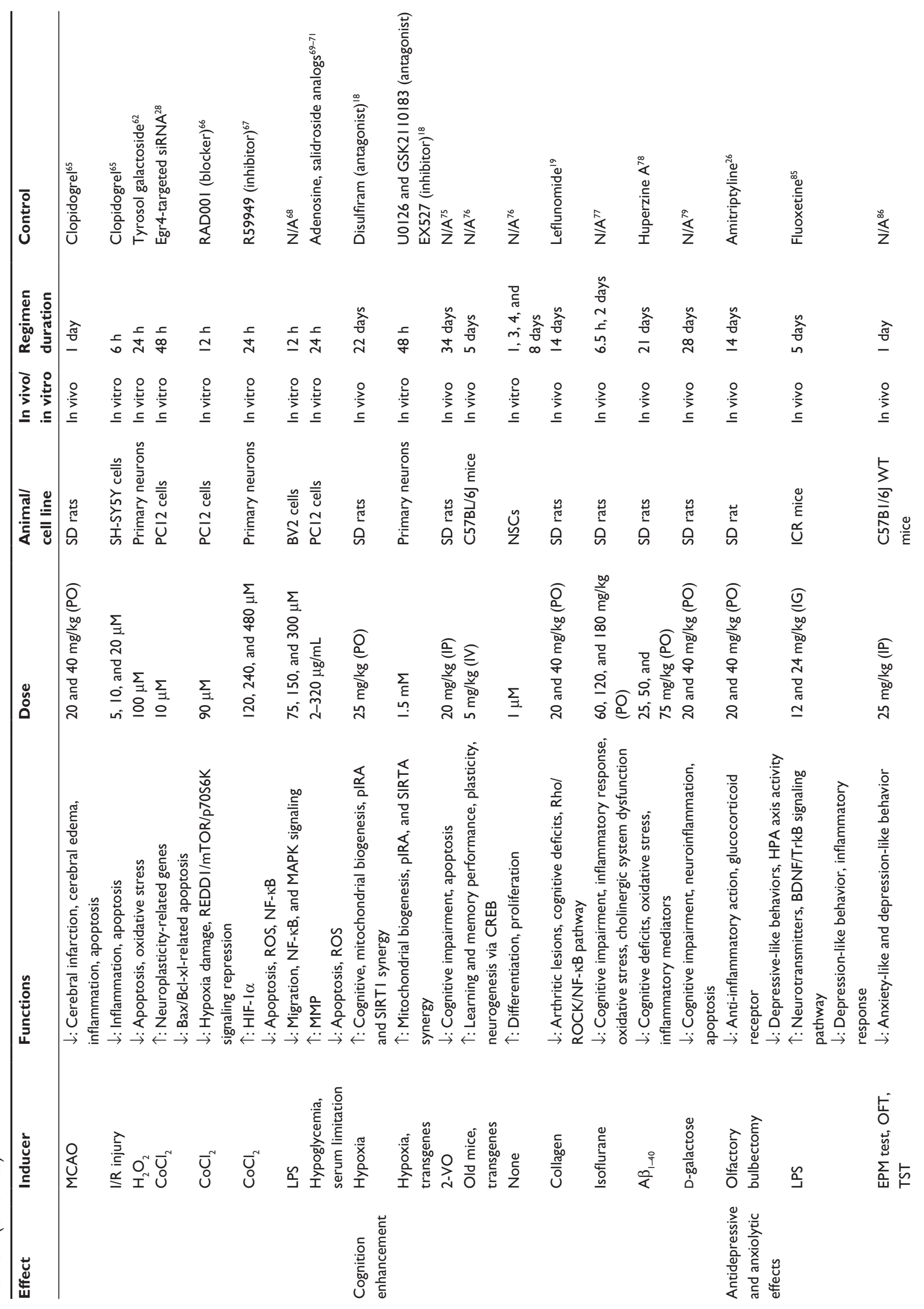


in STZ-induced AD rats by scavenging ROS, improving the proliferation and differentiation of NSCs in vitro..$^{42}$ In a recent study, Gao et $\mathrm{al}^{43}$ showed that salidroside ameliorated D-galactose-induced cognitive deficits in rats with the administration of salidroside (20 and $40 \mathrm{mg} / \mathrm{kg}$ ) once per day for 28 days. It was reported that the mechanism involved in this effect was closely connected with anti-neuroinflammation and apoptosis though mediating inflammatory cytokine levels and apoptosis

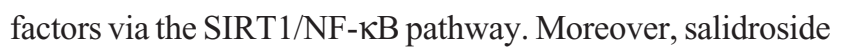
inhibited the excessive increase of $\mathrm{Ca}^{2+}$ and release of calcium stores in $\mathrm{PC} 12$ cells caused by glutamate excitotoxic damage at a dose of $10^{-7}, 10^{-6}$, and $10^{-5} \mathrm{~mol} / \mathrm{L} .{ }^{20} \mathrm{~A}$ further study reported that salidroside $(120$ and $240 \mu \mathrm{M})$ also protected primary hippocampal neurons against glutamate-induced apoptosis via stimulating p-Akt in vitro. ${ }^{44}$ Another study reported that salidroside $(1-100 \mu \mathrm{M})$ regulated MMP, suppressed mitochondrial cyt $c$ release into cytosol, and attenuated caspase activation via an apoptosis pathway in $\mathrm{H}_{2} \mathrm{O}_{2}$-induced $\mathrm{PC} 12$ cells. ${ }^{45}$ Notably, salidroside $(200 \mu \mathrm{M})$ effectively suppressed BACE1 expression, $A \beta$ generation, and $\beta$-secretase activity, and triggered soluble amyloid precursor protein secretion in hypoxia-induced SH-SY5Y cells, suggesting that salidroside may be useful in the prevention and treatment of $\mathrm{AD} .{ }^{46}$

\section{Anti-Parkinson's disease effect}

Parkinson's disease (PD) is the second most common neurodegenerative disorder after $\mathrm{AD}$, and affects about 1\%-2\% of the population over 65 years of age..$^{47,48}$ As a movement disorder, the clinical symptoms of PD include static tremor, rigidity, bradykinesia, and postural instability. L-DOPA is the most effective symptomatic therapy for PD.${ }^{49}$ However, long-term administration of L-DOPA currently causes severe side effects, which has prompted the search of new substitutes for anti-PD treatment. ${ }^{48}$

Li et al and Zhang et al have made notable contributions on the anti-PD effect of salidroside. In their previous studies, salidroside $(1-100 \mu \mathrm{M})$ was reported to inhibit apoptosis, as well as to attenuate MMP collapse, chromatin condensation, and the release of lactate dehydrogenase, induced by $\mathrm{MPP}^{+}(500 \mu \mathrm{M})$ in PC12 cells via NO and the PI3K/Akt pathway. ${ }^{50,51} \mathrm{~A}$ further study indicated that pretreatment with salidroside (15 and $45 \mathrm{mg} / \mathrm{kg}$ ) improved behavior disorders in an MPTP-induced PD mouse model when administered once a day for 7 consecutive days. ${ }^{29,52}$ In these studies, salidroside ameliorated tyrosine hydroxylase-positive neuron loss in SNpc by increasing monoamine substances levels, and the neuroprotection observed may be related to the PI3K/Akt/GSK3 $\beta$ and ROS-NO-related pathways. ${ }^{29,52}$ 


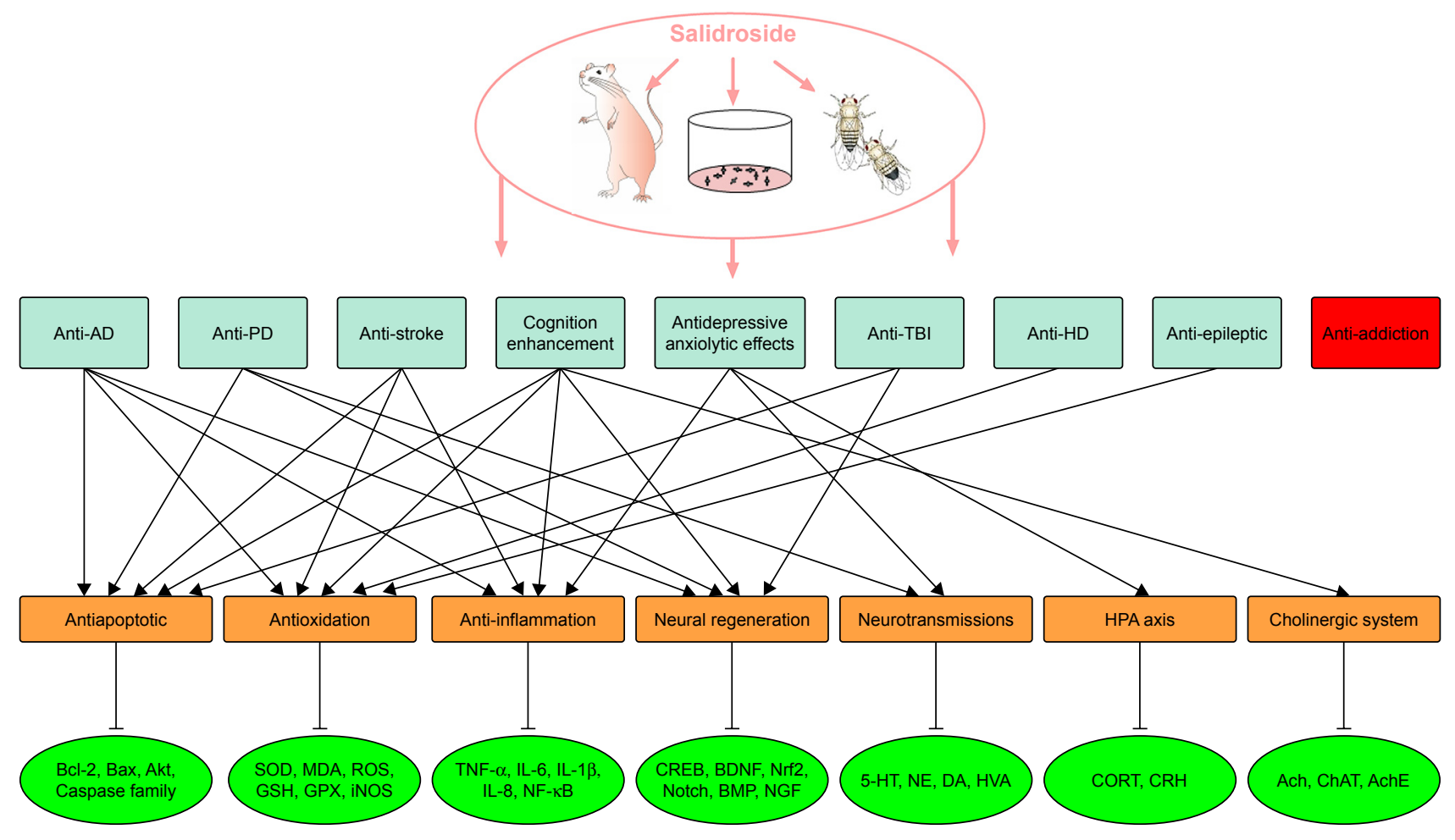

Figure 3 Schematic representation of the possible mechanisms underlying the neuroprotective role of salidroside.

Abbreviations: 5-HT, 5-hydroxytryptamine; Ach, acetylcholine; AchE, acetylcholinesterase; AD, Alzheimer's disease; BDNF, brain-derived neurotrophic factor; BMP, bone morphogenetic protein; ChAT, choline acetyltransferase; CREB, cAMP response element binding protein; GPX, glutathione peroxidase; GSH, glutathione; HD, Huntington's disease; HPA, hypothalamus-pituitary-adrenal; HVA, homovanillic acid; IL-I $\beta$, interleukin-I $\beta$; IL-6, interleukin-6; IL-8, interleukin-8; iNOS, inducible nitric oxide synthase; MDA, malondialdehyde; NE, norephinephrine; NF- $\kappa B$, nuclear factor kappa B; NGF, nerve growth factor; Nrf2, nuclear factor erythroid 2-related factor 2; NSCs, neural stem cells; PD, Parkinson's disease; ROS, reactive oxygen species; SOD, superoxide dismutase; TBI, traumatic brain injury; TNF- $\alpha$, tumor necrosis factor- $\alpha$.

More recently, salidroside $(25-100 \mu \mathrm{M})$ was also reported to significantly reduce $\mathrm{MPP}^{+}$-induced neuronal injury via DJ-1 -Nrf2 antioxidant pathway in SH-SY5Y cells. ${ }^{53}$ Besides, it was demonstrated that salidroside $(100 \mu \mathrm{g} / \mathrm{mL})$ could induce the differentiation of rat mesenchymal stem cells (MSCs) to dopaminergic neurons with increased DA release. ${ }^{54}$ Salidroside was also reported to protect primary cortical neurons and SN4741 cells from endoplasmic reticulum (ER) stress. ${ }^{55}$

\section{Anti-stroke effect}

Stroke is currently the second most common cause of mortality in clinical practice and the third most common cause of disability worldwide, as reported in 2010.56,57 To date, tissue-type plasminogen activator is the only drug approved by the US Food and Drug Administration for the treatment of ischemic stroke. ${ }^{58}$ However, this agent is limited by a narrow therapeutic time window of time and side effects. ${ }^{59}$ Therefore, new neuroprotective agents for this devastating disease are urgently needed.

Zou et $\mathrm{al}^{60}$ initially showed that salidroside-pretreatment had neuroprotective effects on global cerebral ischemiareperfusion rats. In this study, salidroside improved neurological severity scores and reduced the degree of cerebral edema at a dose of $12 \mathrm{mg} / \mathrm{kg}$, whereas the underlying mechanism of action was a possible contribution to free radical scavenging. More recently, it was also reported that salidroside reduced brain edema in focal cerebral ischemia-reperfusion rats with repeated administration of salidroside at a dose of $24 \mathrm{mg} / \mathrm{kg}$ for 7 days by suppressing TNF- $\alpha$ release. ${ }^{61}$ Furthermore, salidroside treatment $(12 \mathrm{mg} / \mathrm{kg})$ once daily for 7 consecutive days before middle cerebral artery occlusion (MCAO) surgery markedly ameliorated neurological scores, and reduced the infarction volume and edema volume in the brain by reversing the decreased normal cells in the hippocampus and the prefrontal cortex. ${ }^{62}$ These results are in agreement with the findings of our team, reporting that salidroside exhibited a relatively strong neuroprotective action against MCAO-induced cerebral ischemia injury, as observed by the neurological deficit scores and infarct area, with administration once daily for 1 day, 2 days, and 6 days $(50 \mathrm{mg} / \mathrm{kg}$, beginning treatment at $1 \mathrm{~h}$ after the operation), or twice (15 and $30 \mathrm{mg} / \mathrm{kg}$, once immediately before cerebral ischemia and once after reperfusion) administration, the underlying mechanisms were due to inhibition of apoptosis (inducting 
the expression of Egrs proteins) and complement drives in Egrs, while salidroside was also related with the activation of the Nrf2/antioxidant response element..$^{28,63,64}$ In a recent study, salidroside $(7.5,20$, and $40 \mathrm{mg} / \mathrm{kg}$, or 5-20 $\mu \mathrm{M})$ significantly inhibited RIP140/nuclear factor (NF)- $\mathrm{KB}$-mediated inflammation and apoptosis by reducing the protein expression ratio of Bax/Bcl-2, and downregulating inflammatory cytokine levels in MCAO rats or I/R injury-caused SH-SY5Y cells; interestingly, the neuroprotective effects of salidroside were comparable with clopidogrel $(10 \mu \mathrm{M})$ on cerebral ischemia, both in vivo and in vitro. ${ }^{65}$

Salidroside had potential applications in cerebral ischemic disease as reported in $\mathrm{H}_{2} \mathrm{O}_{2}, \mathrm{CoCl}_{2}$, glutamate, lipopolysaccharide (LPS), hypoglycemia, glucose, and serum depletioninduced cell models in vitro. A previous study suggested that salidroside exhibited notable neuroprotective actions against $\mathrm{H}_{2} \mathrm{O}_{2}$-induced apoptosis and necrosis by antioxidant activities in primary cultured rat cortical neurons. ${ }^{62}$ Besides, our team further investigated the use of $\mathrm{CoCl}_{2}$-induced hypoxia damage in PC12 cells, showing that the effect of salidroside involved both the mTOR signaling pathway and $\mathrm{Bax} / \mathrm{Bcl}-\mathrm{xL}-$ related apoptosis though the induction of Egrs in vitro. ${ }^{28,66}$ Salidroside $(120,240$, and $480 \mu \mathrm{M})$ also dosedependently inhibited the $\mathrm{CoCl}_{2}$-induced primary cultured cortical neuron apoptosis by enhancing HIF-1 $\alpha$ expression, alleviating ROS level increase and inhibiting NF- $\kappa$ B protein, respectively. ${ }^{67}$ Furthermore, our team found that salidroside (70-300 $\mu \mathrm{M})$ regulated LPS-induced migration in BV2 cells by activating NF- $\mathrm{BB}$, blocking p-MAPK levels (JNK, p38, and ERK1/2), and degrading tropomyosin-related kinase $B$ $(\mathrm{I} \kappa \mathrm{B} \alpha){ }^{68} \mathrm{In}$ addition, several studies suggested that salidroside $(20-320 \mu \mathrm{g} / \mathrm{mL})$ had potential applications in cerebral ischemic disease in hypoglycemia, glucose, and serum depletion-induced models in PC12 cells in a dose-dependent manner. ${ }^{69-71}$ The protective functions of salidroside were mainly related to the attenuation of cytotoxicity by the inhibition of apoptosis and intracellular ROS production, as well as the restoration of MMP. ${ }^{69-71}$

\section{Cognition enhancement}

Learning and memory play an important role in intelligence, while learning and memory impairment is a frequent symptom of attention deficit hyperactivity disorder and fatigue in childhood and adolescent chorea. Such deficits not only negatively affect the patients' quality of life, but are also a heavy burden to society. In recent years, various natural products have been applied for the intervention of these symptoms. $^{72-74}$
It was demonstrated that phosphorylation of insulin receptor subunit A (pIRA) and SIRT1 cooperated to improve cognitive function by mitochondrial biogenesis with repeated administration of salidroside $(25 \mathrm{mg} / \mathrm{kg})$ in hypoxic rats. ${ }^{18}$ Salidroside has also shown protective effects in behavior tests and hippocampal long-term potentiation tests in rats with cognitive deficits caused by chronic cerebral hypoperfusion, by inhibiting apoptosis with administration of salidroside (20 mg/kg) once a day for 34 consecutive days. ${ }^{75}$ These observations were in line with the findings of another study demonstrating that salidroside was effective against learning and memory impairment by stimulating neurogenesis via cAMP response element binding protein (CREB) in the dentate gyrus of aging mice. ${ }^{76} \mathrm{~A}$ further study showed that oral administration of salidroside ameliorated arthritis-induced cognition dysfunction at a dose of 20 and $40 \mathrm{mg} / \mathrm{kg}$ for a consecutive 2 weeks, as observed using a Morris water maze test. According to this study, salidroside significantly decreased pro-inflammatory cytokines though the Rho/ROCK/NF- $\mathrm{BB}$ signaling pathway. ${ }^{19}$ These results were similar to another study reporting that salidroside exerted a protective effect against isoflurane-induced cognitive impairment in behavioral tests when administered at the doses of 60,120 , and $180 \mathrm{mg} / \mathrm{kg}$, and it was suggested that the underlying mechanism of the effect of salidroside was related to inhibition of excessive inflammatory response, decrease of oxidative stress, and regulation of the cholinergic system. ${ }^{77}$ Additionally, salidroside (50 and $75 \mathrm{mg} / \mathrm{kg}$ ) administered once per day for 21 days markedly improved the learning and memory performance in a $A \beta_{1-40}$-injected $A D$ rat model via exerting anti-oxidative and anti-inflammatory properties. ${ }^{78}$ Furthermore, the ameliorating effect of salidroside (20 and $40 \mathrm{mg} / \mathrm{kg}$ for 28 days) on cognitive function may be related to inflammatory cytokines and the SIRT1/NF-кB signaling pathway. ${ }^{79}$

\section{Anti-depressive and anxiolytic effects}

Depression is a serious and common mental disorder characterized by a persistent and prevalent depressed mood. ${ }^{80}$ According to the World Health Organization, depression is currently the fourth most common cause of morbidity and the second common cause of disability worldwide. ${ }^{81-83}$ Depression also tremendously increases the risk of suicide attempts and suicide. ${ }^{84}$ Pharmacotherapy remains at the core of treatment for depression. Although various antidepressants are currently available for the treatment of this disorder, the majority of these agents take a long time to take into full effect, while many of these usually result in co-morbid symptoms and a poor tolerance. ${ }^{80}$ Therefore, there is a clear 
need for new targets and a therapeutic candidate with higher efficacy and lower toxicity.

It was reported that chronic treatment with salidroside (20 and $40 \mathrm{mg} / \mathrm{kg}$ ) exhibited an antidepressant-like activity in olfactory bulbectomized rats in behavior tests, and that salidroside activity was comparable with that of amitriptyline $(10 \mathrm{mg} / \mathrm{kg}){ }^{76}$ This previous study also demonstrated that the antidepressant-like effect of salidroside might be correlated to its anti-inflammatory effects and the regulation of hypothalamus-pituitary-adrenal (HPA) axis activity by reversing ER abnormalities. ${ }^{26}$ Similarly, salidroside was also reported to affect depression-like behaviors in LPS-induced mice at a dose of 12 and $24 \mathrm{mg} / \mathrm{kg}$ once daily for 5 consecutive days, and the results also indicated that the antidepressant mechanism of salidroside was related to brain-derived neurotrophic factor/tropomyosin-related kinase B (BDNF/ TrkB) signaling through increasing neurotransmitters and decreasing pro-inflammatory cytokines. ${ }^{85}$ More importantly, Palmeri et $\mathrm{al}^{86}$ observed that acute administration of salidroside $(25 \mathrm{mg} / \mathrm{kg})$ exhibited antidepressant and anxiolytic effects with a high therapeutic efficacy and a low spectrum of adverse effects in mice.

\section{Amelioration of traumatic brain injury}

Salidroside treatment also led to better behavior performance in contusion-induced traumatic brain injury (TBI) in mice at a dose of 20 and $50 \mathrm{mg} / \mathrm{kg}$ by suppressing PI3K/Akt signaling pathway-mediated apoptosis. ${ }^{22}$ Furthermore, it was evidenced that salidroside $(5-100 \mu \mathrm{g} / \mathrm{mL})$ promoted the differentiation of MSCs D1 cells through mediating the Notch and BMP signaling pathway. ${ }^{87}$

\section{Anti-Huntington's disease effect}

In N2, HA759, and AM141 nematodes, Xiao et al ${ }^{24}$ observed that salidroside (50-200 $\mu \mathrm{M})$ exhibited a neuroprotective effect via the oxidative stress pathway against polyQ toxicity, suggesting that salidroside could serve as a useful anti-HD agent.

\section{Anti-addiction effect}

It was reported that treatment of male CD-1 mice with $0.2 \mathrm{mg} / \mathrm{kg}$ salidroside lowered the rewarding properties and prevented relapse to nicotine. ${ }^{23}$ These findings provide experimental foundation for further research on salidroside as a drug for withdrawal from nicotine addiction.

\section{Anti-epileptic effect}

Si et $\mathrm{al}^{31}$ researched the effect of salidroside $(25$ and $50 \mathrm{mg} / \mathrm{kg}$ ) on kainic acid-induced SE in C57BL/6 mice, and found that salidroside pretreatment only once significantly increased the latency to epileptic and reduced the incidence of epileptic via suppressing oxidative stress. Furthermore, the AMPK/SIRT1/ FoxO1 signaling pathway is possibly activated by salidroside.

\section{Safety}

Salidroside is commonly considered to be a safe and effective substance. It has been concluded that salidroside does not result in maternal or embryonic toxicity, and there are no teratogenic effects under the experimental conditions at a dose of $0.5,0.25$, and $0.125 \mathrm{~g} / \mathrm{kg}$ in SD rats. ${ }^{88}$ Genotoxicity evaluation is essential in the risk assessment of drugs. The results of Ames test, reverse mutation assay, chromosomal aberrations assay, and mouse micronucleus assay have shown that salidroside is not genotoxic at a clinical dose $(150 \mathrm{mg} /$ $60 \mathrm{~kg} /$ day) for humans. ${ }^{89,90}$ Another randomized and controlled trial of 60 breast cancer cases showed that there were no clinical adverse events when an effective dose of salidroside (600 mg/kg/day) was administered during the entire therapeutic process. ${ }^{91}$ Thus, the lack of adverse effects in the course of pre-clinical and clinical trials indicate that salidroside is promising as a common clinical drug.

\section{Clinical studies}

Some publications on clinical efficacy have demonstrated that Rhodiola extracts are helpful for learning and memory, especially concentration, and that they are extremely helpful for patients with mild-to-moderate depression. ${ }^{92-97}$ Rhodiola extract injection and Rhodiola capsules are widely used for stroke rehabilitation and to treat high-altitude hypoxia clinically. Although many studies have demonstrated clear neuroprotective effects of salidroside in cell lines and/or rat models, none of the salidroside preparations have so far entered medical clinical trials, and there has been no clinical evidence to confirm the efficacy of salidroside. Therefore, highly qualified and large controlled clinical experiments are expected in order to better direct the clinical application of salidroside.

\section{Conclusion}

This review article extensively summarized several studies in order to determine the neuropharmacological role of salidroside and its possible underlying mechanisms. According to these studies, salidroside possesses various biological activities in the nervous system, including anti-AD, anti-PD, anti-stroke, cognitive function improvement, mood amelioration, anti-HD, anti-aging, anti-addiction, and anti-epileptic effects. Salidroside has powerful bioactive effects and can regulate oxidative stress, inflammation, apoptosis, the HPA axis, neurotransmission, the cholinergic system, and neural 
regeneration. Although the function of salidroside in the central nervous system has been investigated in preclinical tests, no data are available that can provide safe and effective clinical evidence in patients, and definite target protein conjugates for salidroside are still lacking. Therefore, as a bioactive compound of traditional Chinese medicine, the applications and efficacy of salidroside in central nervous system diseases require further investigation.

\section{Acknowledgments}

This work was supported by the research project of Fujian Provincial Health and Family Planning Commission (20171-80), the project of Fujian Province Office of Education (JZ160442), and Fujian Provincial Science and Technology Department (2018R1035-9), and Fujian Provincial Science and Technology Department (2018R1035-11).

\section{Author contributions}

All authors contributed toward data analysis, drafting and critically revising the paper, gave final approval of the version to be published, and agree to be accountable for all aspects of the work.

\section{Disclosure}

The authors report no conflicts of interest in this work.

\section{References}

1. Nabavi SF, Braidy N, Orhan IE, Badiee A, Daglia M, Nabavi SM. Rhodiola rosea L. and Alzheimer's disease: from farm to pharmacy. Phytother Res. 2016;30(4):532-539.

2. Panossian A, Wikman G, Sarris J. Rosenroot (Rhodiola rosea): traditional use, chemical composition, pharmacology and clinical efficacy Phytomedicine. 2010;17(7):481-493.

3. Kelly GS. Rhodiola rosea: a possible plant adaptogen. Altern Med Rev. 2001;6(3):293-302.

4. Perfumi M, Mattioli L. Adaptogenic and central nervous system effects of single doses of 3\% rosavin and $1 \%$ salidroside Rhodiola rosea $\mathrm{L}$. extract in mice. Phytother Res. 2007;21(1):37-43.

5. Yousef GG, Grace MH, Cheng DM, Belolipov IV, Raskin I, Lila MA. Comparative phytochemical characterization of three Rhodiola species. Phytochemistry. 2006;67(21):2380-2391.

6. Shi L, Ma Y, Cai Z. Quantitative determination of salidroside and specnuezhenide in the fruits of Ligustrum lucidum Ait by high performance liquid chromatography. Biomed Chromatogr. 1998;12(1):27-30.

7. Li YR, Li C, Wang ZM, Yang LX. [Chemical constituents from whole plants of Aconitum tanguticum (III)]. Zhongguo Zhong Yao Za Zhi. 2014;39(7):1163-1167. Chinese.

8. Xu L, Li YR, Li C, Lin LM, Wang ZM, Luo YM. [Chemical constituents from whole plants of Aconitum tanguticum]. Zhongguo Zhong Yao Za Zhi. 2013;38(17):2818-2825. Chinese.

9. Cui S, Hu X, Chen X, Hu Z. Determination of p-tyrosol and salidroside in three samples of Rhodiola crenulata and one of Rhodiola kirilowii by capillary zone electrophoresis. Anal Bioanal Chem. 2003; 377(2):370-374

10. Cui JL, Wang YN, Jiao J, Gong Y, Wang JH, Wang ML. Fungal endophyte-induced salidroside and tyrosol biosynthesis combined with signal cross-talk and the mechanism of enzyme gene expression in Rhodiola crenulata. Sci Rep. 2017;7(1):12540.
11. Yu HL, Xu JH, Su JH, Lu WY, Lin GQ. Synthesis of novel salidroside esters by lipase-mediated acylation with various functional acyl groups. J Biosci Bioeng. 2008;106(1):65-68.

12. Guo Y, Zhao Y, Zheng C, Meng Y, Yang Y. Synthesis, biological activity of salidroside and its analogues. Chem Pharm Bull (Tokyo). 2010; 58(12):1627-1629.

13. Li Y, Lian ML, Shao CH, Jin C, Piao XC. [Effect of methyl jasmonate on salidroside and polysaccharide accumulation in Rhodiola sachalinensis callus]. Zhongguo Zhong Yao Za Zhi. 2014;39(21):4252-4257. Chinese.

14. Wang Y-W, Hu G-S, Jia J-M. Effects of methyl jasmonate and 2-aminoindan-2-phosphate treatment on growth and salidroside accumulation in callus culture of Rhodiola sachalinensis A. Bor. J Shenyang Pharm Uni. 2012;29(9):718-723.

15. Chung D, Kim SY, Ahn JH. Production of three phenylethanoids, tyrosol, hydroxytyrosol, and salidroside, using plant genes expressing in Escherichia coli. Sci Rep. 2017;7(1):2578.

16. Fan B, Chen T, Zhang S, Wu B, He B. Author correction: Mining of efficient microbial UDP-glycosyltransferases by motif evolution cross plant kingdom for application in biosynthesis of salidroside. Sci Rep. 2017;7(1): 17147.

17. Guo N, Hu Z, Fan X, et al. Simultaneous determination of salidroside and its aglycone metabolite p-tyrosol in rat plasma by liquid chromatography-tandem mass spectrometry. Molecules. 2012;17(4):4733-4754.

18. Barhwal K, Das SK, Kumar A, Hota SK, Srivastava RB. Insulin receptor A and Sirtuin 1 synergistically improve learning and spatial memory following chronic salidroside treatment during hypoxia. J Neurochem. 2015;135(2):332-346.

19. Zhu L, Chen T, Chang X, et al. Salidroside ameliorates arthritis-induced brain cognition deficits by regulating Rho/ROCK/NF-KB pathway. Neuropharmacology. 2016;103:134-142.

20. Cao LL, Du GH, Wang MW. The effect of salidroside on cell damage induced by glutamate and intracellular free calcium in PC12 cells. $J$ Asian Nat Prod Res. 2006;8(1-2):159-165.

21. Wang H, Ding Y, Zhou J, Sun X, Wang S. The in vitro and in vivo antiviral effects of salidroside from Rhodiola rosea L. against coxsackievirus B3. Phytomedicine. 2009;16(2-3):146-155.

22. Chen SF, Tsai HJ, Hung TH, et al. Salidroside improves behavioral and histological outcomes and reduces apoptosis via PI3K/Akt signaling after experimental traumatic brain injury. PLoS One. 2012;7(9):e45763.

23. Titomanlio F, Perfumi M, Mattioli L. Rhodiola rosea L. extract and its active compound salidroside antagonized both induction and reinstatement of nicotine place preference in mice. Psychopharmacology. 2014;231(10):2077-2086.

24. Xiao L, Li H, Zhang J, et al. Salidroside protects Caenorhabditis elegans neurons from polyglutamine-mediated toxicity by reducing oxidative stress. Molecules. 2014;19(6):7757-7769.

25. Xing S, Yang X, Li W, et al. Salidroside stimulates mitochondrial biogenesis and protects against $\mathrm{H}_{2} \mathrm{O}_{2}$-induced endothelial dysfunction. Oxid Med Cell Longev. 2014;2014:904834.

26. Yang SJ, Yu HY, Kang DY, et al. Antidepressant-like effects of salidroside on olfactory bulbectomy-induced pro-inflammatory cytokine production and hyperactivity of HPA axis in rats. Pharmacol Biochem Behav. 2014;124:451-457.

27. Grech-Baran M, Syklowska-Baranek K, Pietrosiuk A. Biotechnological approaches to enhance salidroside, rosin and its derivatives production in selected Rhodiola spp. in vitro cultures. Phytochem Rev. 2015; 14(4):657-674.

28. Lai W, Zheng Z, Zhang X, et al. Salidroside-mediated neuroprotection is associated with induction of early growth response genes (Egrs) across a wide therapeutic window. Neurotox Res. 2015;28(2):108-121.

29. Wang S, He H, Chen L, Zhang W, Zhang X, Chen J. Protective effects of salidroside in the MPTP/MPP(+)-induced model of Parkinson's disease through ROS-NO-related mitochondrion pathway. Mol Neurobiol. 2015;51(2):718-728.

30. Xing SS, Yang XY, Zheng T, et al. Salidroside improves endothelial function and alleviates atherosclerosis by activating a mitochondriarelated AMPK/PI3K/Akt/eNOS pathway. Vascul Pharmacol. 2015;72: $141-152$. 
31. Si PP, Zhen JL, Cai YL, Wang WJ, Wang WP. Salidroside protects against kainic acid-induced status epilepticus via suppressing oxidative stress. Neurosci Lett. 2016;618:19-24.

32. Wu D, Yang X, Zheng T, et al. A novel mechanism of action for salidroside to alleviate diabetic albuminuria: effects on albumin transcytosis across glomerular endothelial cells. Am J Physiol Endocrinol Metab. 2016;310(3):E225-E237.

33. Berchtold NC, Cotman CW. Evolution in the conceptualization of dementia and Alzheimer's disease: Greco-Roman period to the 1960s. Neurobiol Aging. 1998;19(3):173-189.

34. Wimo A, Jonsson L, Bond J, Prince M, Winblad B. Alzheimer Disease International. The worldwide economic impact of dementia 2010. Alzheimers Dement. 2013;9(1):1.e13-11.e13.

35. Rossignol DA, Frye RE. The use of medications approved for Alzheimer's disease in autism spectrum disorder: a systematic review. Fronti Pediatr. 2014;2:87.

36. Yang G, Wang Y, Tian J, Liu JP. Huperzine A for Alzheimer's disease: a systematic review and meta-analysis of randomized clinical trials. PLoS One. 2013;8(9):e74916.

37. Maccioni RB, Munoz JP, Barbeito L. The molecular bases of Alzheimer's disease and other neurodegenerative disorders. Arch Med Res. 2001;32(5):367-381.

38. Jang SI, Pae HO, Choi BM, et al. Salidroside from Rhodiola sachalinensis protects neuronal PC12 cells against cytotoxicity induced by amyloid-beta. Immunopharmacol Immunotoxicol. 2003;25(3):295-304.

39. Zhang L, Yu H, Zhao X, et al. Neuroprotective effects of salidroside against beta-amyloid-induced oxidative stress in SH-SY5Y human neuroblastoma cells. Neurochem Int. 2010;57(5):547-555.

40. Zhang B, Wang Y, Li H, et al. Neuroprotective effects of salidroside through PI3K/Akt pathway activation in Alzheimer's disease models. Drug Des Devel Ther. 2016;10:1335-1343.

41. Zhang B, Li Q, Chu X, Sun S, Chen S. Salidroside reduces tau hyperphosphorylation via up-regulating GSK- $3 \beta$ phosphorylation in a tau transgenic Drosophila model of Alzheimer's disease. Transl Neurodegener. 2016;5:21.

42. Qu ZQ, Zhou Y, Zeng YS, et al. Protective effects of a Rhodiola crenulata extract and salidroside on hippocampal neurogenesis against streptozotocin-induced neural injury in the rat. PLoS One. 2012;7(1): e29641.

43. Gao J, Zhou R, You X, et al. Salidroside suppresses inflammation in a D-galactose-induced rat model of Alzheimer's disease via SIRT1/

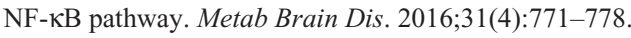

44. Xian $\mathrm{H}$, Zhao J, Zheng Y, et al. MADP, a salidroside analog, protects hippocampal neurons from glutamate induced apoptosis. Life Sci. 2014;103(1):34-40.

45. Cai L, Wang H, Li Q, Qian Y, Yao W. Salidroside inhibits H2O2induced apoptosis in PC12 cells by preventing cytochrome c release and inactivating of caspase cascade. Acta Biochim Biophys Sin. 2008; 40(9):796-802.

46. Li QY, Wang HM, Wang ZQ, Ma JF, Ding JQ, Chen SD. Salidroside attenuates hypoxia-induced abnormal processing of amyloid precursor protein by decreasing BACE1 expression in SH-SY5Y cells. Neurosci Lett. 2010;481(3):154-158.

47. Ferreira M, Massano J. An updated review of Parkinson's disease genetics and clinicopathological correlations. Acta Neurol Scand. 2017; 135(3):273-284.

48. Cacabelos R. Parkinson's disease: from pathogenesis to pharmacogenomics. Int J Mol Sci. 2017;18(3):pii:E551.

49. Katzenschlager R, Lees AJ. Treatment of Parkinson's disease: levodopa as the first choice. J Neurol. 2002;249(Suppl 2):II19-II24.

50. Li X, Ye X, Li X, et al. Salidroside protects against MPP(+)-induced apoptosis in PC12 cells by inhibiting the NO pathway. Brain Res. 2011; 1382:9-18.

51. Zhang L, Ding W, Sun H, et al. Salidroside protects PC12 cells from MPP(+)-induced apoptosis via activation of the PI3K/Akt pathway. Food Chem Toxicol. 2012;50(8):2591-2597.
52. Zhang W, He H, Song H, et al. Neuroprotective effects of salidroside in the MPTP mouse model of Parkinson's disease: involvement of the PI3K/Akt/GSK3ß pathway. Parkinson's Dis. 2016;2016:9450137.

53. Wu L, Xu H, Cao L, et al. Salidroside protects against MPP+-induced neuronal injury through DJ-1-Nrf2 antioxidant pathway. Evid Based Complement Alternat Med. 2017;2017:5398542.

54. Zhao HB, Ma H, Ha XQ, et al. Salidroside induces rat mesenchymal stem cells to differentiate into dopaminergic neurons. Cell Biol Int. 2014;38(4):462-471.

55. Tao K, Wang B, Feng D, et al. Salidroside protects against 6-hydroxydopamine-induced cytotoxicity by attenuating ER stress. Neurosci Bull. 2016;32(1):61-69.

56. Lozano R, Naghavi M, Foreman K, et al. Global and regional mortality from 235 causes of death for 20 age groups in 1990 and 2010: a systematic analysis for the Global Burden of Disease Study 2010. Lancet. 2012;380(9859):2095-2128.

57. Murray CJ, Vos T, Lozano R, et al. Disability-adjusted life years (DALYs) for 291 diseases and injuries in 21 regions, 1990-2010: a systematic analysis for the Global Burden of Disease Study 2010. Lancet. 2012;380(9859):2197-2223.

58. Zivin JA. Acute stroke therapy with tissue plasminogen activator (tPA) since it was approved by the U.S. Food and Drug Administration (FDA). Ann Neurol. 2009;66(1):6-10.

59. Davis S, Lees K, Donnan G. Treating the acute stroke patient as an emergency: current practices and future opportunities. Int J Clin Pract. 2006;60(4):399-407.

60. Zou YQ, Cai ZY, Mao YF, Li JB, Deng XM. [Effects of salidrosidepretreatment on neuroethology of rats after global cerebral ischemiareperfusion]. Zhong Xi Yi Jie He Xue Bao. 2009;7(2):130-134.

61. Han T. Effects of salidroside pretreatment on expression of tumor necrosis factor-alpha and permeability of blood brain barrier in rat model of focal cerebralischemia-reperfusion injury. Asian Pac J Trop Med. 2013;6(2):156-158.

62. Shi TY, Feng SF, Xing JH, et al. Neuroprotective effects of Salidroside and its analogue tyrosol galactoside against focal cerebral ischemia in vivo and $\mathrm{H} 2 \mathrm{O} 2$-induced neurotoxicity in vitro. Neurotox Res. 2012; 21(4):358-367.

63. Han J, Xiao Q, Lin YH, et al. Neuroprotective effects of salidroside on focal cerebral ischemia/reperfusion injury involve the nuclear erythroid 2-related factor 2 pathway. Neural Regen Res. 2015;10(12): 1989-1996.

64. Lai W, Xie X, Zhang X, et al. Inhibition of complement drives increase in early growth response proteins and neuroprotection mediated by salidroside after cerebral ischemia. Inflammation. 2018;41(2):449-463.

65. Chen T, Ma Z, Zhu L, et al. Suppressing receptor-interacting protein 140: a new sight for salidroside to treat cerebral ischemia. Mol Neurobiol. 2016;53(9):6240-6250.

66. Zhong X, Lin R, Li Z, Mao J, Chen L. Effects of salidroside on cobalt chloride-induced hypoxia damage and mTOR signaling repression in PC12 cells. Biol Pharm Bull. 2014;37(7):1199-1206.

67. Zhang S, Chen X, Yang Y, Zhou X, Liu J, Ding F. Neuroprotection against cobalt chloride-induced cell apoptosis of primary cultured cortical neurons by salidroside. Mol Cell Biochem. 2011;354(1-2):161-170.

68. Hu H, Li Z, Zhu X, Lin R, Chen L. Salidroside reduces cell mobility via NF- $\mathrm{KB}$ and MAPK signaling in LPS-induced BV2 microglial cells. Evid Based Complement Alternat Med. 2014;2014:383821.

69. Yu S, Liu M, Gu X, Ding F. Neuroprotective effects of salidroside in the PC12 cell model exposed to hypoglycemia and serum limitation. Cell Mol Neurobiol. 2008;28(8):1067-1078.

70. Yang X, Xu W, Zhao W, Zhao Y, Yang Y, Ling Y. Synthesis and neuroprotective effects of the fluorine substituted salidroside analogues in the PC12 cell model exposed to hypoglycemia and serum limitation. Chem Pharm Bull (Tokyo). 2013;61(11):1192-1196.

71. Zhao Y, Ling Y, Zhao J, et al. Synthesis and protective effects of novel salidroside analogues on glucose and serum depletion induced apoptosis in PC12 cells. Arch Pharm (Weinheim). 2013;346(4):300-307. 
72. Zhang H, Lai Q, Li Y, Liu Y, Yang M. Learning and memory improvement and neuroprotection of Gardenia jasminoides (Fructus gardenia) extract on ischemic brain injury rats. J Ethnopharmacol. 2017;196: 225-235.

73. Asadi-Shekaari M, Karimi A, Shabani M, Sheibani V, Esmaeilpour K. Maternal feeding with walnuts (Juglans regia) improves learning and memory in their adult pups. Avicenna J Phytomed. 2013;3(4): 341-346.

74. Rabiei Z, Rafieian-Kopaei M, Heidarian E, Saghaei E, Mokhtari S. Effects of Zizyphus jujube extract on memory and learning impairment induced by bilateral electric lesions of the nucleus Basalis of Meynert in rat. Neurochem Res. 2014;39(2):353-360.

75. Yan ZQ, Chen J, Xing GX, Huang JG, Hou XH, Zhang Y. Salidroside prevents cognitive impairment induced by chronic cerebral hypoperfusion in rats. $J$ Int Med Res. 2015;43(3):402-411.

76. Jin H, Pei L, Shu X, et al. Therapeutic intervention of learning and memory decays by salidroside stimulation of neurogenesis in aging. Mol Neurobiol. 2016;53(2):851-866.

77. Liang L, Ma Z, Dong M, Ma J, Jiang A, Sun X. Protective effects of salidroside against isoflurane-induced cognitive impairment in rats. Hum Exp Toxicol. 2017;36(12):1295-1302.

78. Zhang J, Zhen YF, Pu-Bu-Ci-Ren, et al. Salidroside attenuates beta amyloid-induced cognitive deficits via modulating oxidative stress and inflammatory mediators in rat hippocampus. Behav Brain Res. 2013 244:70-81.

79. Gao J, He H, Jiang W, et al. Salidroside ameliorates cognitive impairment in a d-galactose-induced rat model of Alzheimer's disease. Behav Brain Res. 2015;293:27-33.

80. Millan MJ. Multi-target strategies for the improved treatment of depressive states: conceptual foundations and neuronal substrates, drug discovery and therapeutic application. Pharmacol Ther. 2006; 110(2):135-370.

81. Kessler RC, Berglund P, Demler O, et al. The epidemiology of major depressive disorder: results from the National Comorbidity Survey Replication (NCS-R). JAMA. 2003;289(23):3095-3105.

82. Kessler RC, Ormel J, Petukhova M, et al. Development of lifetime comorbidity in the World Health Organization world mental health surveys. Arch Gen Psychiatry. 2011;68(1):90-100.

83. Global Burden of Disease Study 2013 Collaborators. Global, regional, and national incidence, prevalence, and years lived with disability for 301 acute and chronic diseases and injuries in 188 countries, 1990-2013. a systematic analysis for the Global Burden of Disease Study 2013. Lancet. 2015;386(9995):743-800.

84. Sartorius N, Baghai TC, Baldwin DS, et al. Antidepressant medications and other treatments of depressive disorders: a CINP Task Force report based on a review of evidence. Int J Neuropsychopharmacol. 2007; 10(Suppl 1):S1-S207.
85. Zhu L, Wei T, Gao J, et al. Salidroside attenuates lipopolysaccharide (LPS) induced serum cytokines and depressive-like behavior in mice. Neurosci Lett. 2015;606:1-6.

86. Palmeri A, Mammana L, Tropea MR, Gulisano W, Puzzo D. Salidroside, a bioactive compound of rhodiola rosea, ameliorates memory and emotional behavior in adult mice. J Alzheimers Dis. 2016;52(1):65-75.

87. Zhao HB, Qi SN, Dong JZ, et al. Salidroside induces neuronal differentiation of mouse mesenchymal stem cells through Notch and BMP signaling pathways. Food Chem Toxicol. 2014;71:60-67.

88. Zhu Y-P, Zhu J-B, Ma X-L, Tian Y-J, Wan X-Y, Zhang T-B. [Evaluation for developmental toxicity of rhodioside injection in rats]. Chin J New Drugs. 2009;18(21):2068-2071. Chinese.

89. Zhu J, Wan X, Zhu Y, Ma X, Zheng Y, Zhang T. Evaluation of salidroside in vitro and in vivo genotoxicity. Drug Chem Toxicol. 2010;33(2) 220-226.

90. Zhu Y-P, Zhang T-B, Wan X, et al. Genetic toxicity of rhodioside injection. Pharm Care Res. 2009;9(4):279-282.

91. Zhang H, Shen WS, Gao CH, Deng LC, Shen D. Protective effects of salidroside on epirubicin-induced early left ventricular regional systolic dysfunction in patients with breast cancer. Drugs $R$ D. 2012; 12(2):101-106.

92. Darbinyan V, Aslanyan G, Amroyan E, Gabrielyan E, Malmstrom C, Panossian A. Clinical trial of Rhodiola rosea L. extract SHR-5 in the treatment of mild to moderate depression. Nord J Psychiatry. 2007;61(5) 343-348.

93. Spasov AA, Wikman GK, Mandrikov VB, Mironova IA, Neumoin VV. A double-blind, placebo-controlled pilot study of the stimulating and adaptogenic effect of Rhodiola rosea SHR-5 extract on the fatigue of students caused by stress during an examination period with a repeated low-dose regimen. Phytomedicine. 2000;7(2):85-89.

94. Shevtsov VA, Zholus BI, Shervarly VI, et al. A randomized trial of two different doses of a SHR-5 Rhodiola rosea extract versus placebo and control of capacity for mental work. Phytomedicine. 2003;10(2-3): 95-105.

95. Darbinyan V, Kteyan A, Panossian A, Gabrielian E, Wikman G, Wagner $\mathrm{H}$. Rhodiola rosea in stress induced fatigue - a double blind cross-over study of a standardized extract SHR-5 with a repeated lowdose regimen on the mental performance of healthy physicians during night duty. Phytomedicine. 2000;7(5):365-371.

96. Mao JJ, Li QS, Soeller I, Xie SX, Amsterdam JD. Rhodiola rosea therapy for major depressive disorder: a study protocol for a randomized, double-blind, placebo-controlled trial. J Clin Trials. 2014;4:170.

97. Mao JJ, Xie SX, Zee J, et al. Rhodiola rosea versus sertraline for major depressive disorder: A randomized placebo-controlled trial. Phytomedicine. 2015;22(3):394-399.

\section{Publish your work in this journal}

Drug Design, Development and Therapy is an international, peerreviewed open-access journal that spans the spectrum of drug design and development through to clinical applications. Clinical outcomes, patient safety, and programs for the development and effective, safe, and sustained use of medicines are the features of the journal, which

\section{Dovepress}

has also been accepted for indexing on PubMed Central. The manuscript management system is completely online and includes a very quick and fair peer-review system, which is all easy to use. Visit http://www.dovepress.com/testimonials.php to read real quotes from published authors. 